\title{
ENJEUX DE LA MÉDIATION CORPORELLE AU SEIN DU MÉDIA EXPOSITION
}

\author{
Philippe Verhaegen ${ }^{1}$
}

Les thèses des sciences cognitives nous ont habitués à considérer les processus cognitifs comme manipulant tantôt des symboles et des propositions logiques, tantôt des images et des métaphores. Dans ce dernier cas une certaine place a été accordée à l'expérience vécue du sujet et à sa corporéité. Si l'on peut concevoir, comme l'envisage ce dossier, une pensée iconique, c'est parce qu'elle est sous-tendue par une pensée incarnée, corporelle. C'est du moins ce que les lignes qui suivent tentent de montrer dans le cadre d'un dispositif particulier, celui des musées et des expositions de médiation de savoirs².

La recherche en communication s'est abondamment intéressée à l'analyse des médias et des grands dispositifs d'expression comme le texte, l'image et leurs différentes combinaisons (films, documentaires, journaux,...) mais fort peu d'attention a été accordée à la médiation corporelle. Il existe pourtant des dispositifs de communication qui accordent une place centrale au corps. Les musées, les centres d'interprétation, les expositions sont des lieux où les objets présentés ne sont accessibles qu'au travers d'une démarche de visite impliquant une

1 Professeur à l'Université catholique de Louvain, Groupe de Recherche en Médiation des Savoirs (GReMS).

2 Même si l'optique adoptée dans cet article pourrait leur être appliquée, nous laisserons de côté les musées d'art et de patrimoine qui soulèvent un autre type de questionnement.

Recherches en communication, $\mathrm{n}^{\circ} 29$ (2008). 
participation corporelle active faite de déambulation, d'observation et de manipulation. C'est en effet en butinant d'un exhibit à un autre, en se saisissant d'un objet ou en effectuant une opération manuelle que le visiteur est invité à s'approprier le savoir mis à sa disposition par le concepteur de l'exposition. À côté de ces dispositifs impliquant physiquement les individus, on en trouve de plus en plus qui en proposent une présence virtuelle. Les réseaux informatiques et les technologies qui l'accompagnent démultiplient aujourd'hui la présence d'un même individu sur différents sites (et vice versa). L'usager - l'internaute - est en quelque sorte fixé devant un écran et des interfaces sont mises à sa disposition pour interagir avec l'outil. Quoique sa mobilité soit réduite, sa motricité, elle, est bien réelle que celle-ci passe par le regard ou par les mains prolongées par une souris ou un joystick. Bon nombre de jeux vidéo ou de logiciels interactifs proposent des univers virtuels - visite de ville ou de musées - basés sur la communication par avatars qui forment autant de lieux de simulation corporelle. « La place du corps dans les démarches d'apprentissage n'est certes pas un thème inédit, les environnements interactifs ont renouvelé l'approche des échanges entre le corporel et l'intellect. Avancées que le développement en cours d'interfaces à retour d'effort ne manquera pas encore de prolonger. ${ }^{1}$

Si la place du corps dans un certain nombre de dispositifs est manifeste, celle des difficultés sémiotiques liées à son analyse l'est tout autant. Dans sa recherche des codes, le structuralisme linguistique a produit divers dictionnaires gestuels censés présenter les unités élémentaires d'un hypothétique « langage gestuel ». Certains chercheurs -Kristeva par exemple- avait pourtant mis en garde contre cette dérive : le geste ne doit pas être appréhendé comme un message codé. Il est une production antérieure au message représenté et représentable. Il faut donc l'envisager « comme une activité dans le sens d'une dépense, d'une productivité antérieure au produit, donc antérieure à la représentation comme phénomène de signification dans le circuit communicatif $»^{2}$. On doit donc l'analyser en termes de fonctions. Aujourd'hui les sciences cognitives renouvellent cette problématique. C'est notre insertion corporelle dans le monde qui permet la construction d'images - schémas, c'està-dire de structures simples qui organisent nos expériences concrètes

1 J.L. WEISSBERG, "Retour sur interactivité", Revue des sciences de l'éducation, Vol. XXV, n 1, 1999, p. 172.

2 J.KrISTEVA, "Le geste, pratique ou communication", Revue Langages, n¹0, Didier-Larousse, 1968, p. 50. 
et les rend intelligibles. À partir de celles-ci, des représentations plus complexes peuvent voir le jour. En quelque sorte le corps est dans l'esprit $^{1}$. Reste à voir comment on peut l'appréhender dans le cadre du musée.

Dans leur ethnographie d'une exposition, Veron et Levasseur considèrent que «l'expo est d'abord un lieu et comme tout lieu sa pratique met fondamentalement en jeu le corps : d'une certaine manière, l'itinéraire d'un individu dans une exposition matérialise, inscrit au sol, son itinéraire dans l'information et la sensation. Trajets, stationnements, évitements sont autant d'indicateurs physiquement objectivé, donc facilement observables, d'un parcours culturel et d'une progression perceptive $»^{2}$. Reste toutefois à expliquer le lien entre ces traces corporelles et l'acquisition de savoirs.

Le musée c'est également un lieu d'implication de nos différents sens. Dans le musée traditionnel, seule la vue est convoquée. Les objets, placé derrière des vitrines, s'offre au regard du visiteur qui, toutefois, ne peut -et n'est pas autorisé à- les manipuler. Cela frise parfois le ridicule quand, dans une exposition consacrée aux instruments de musique, le visiteur ne peut toucher les instruments exposés mais a juste la possibilité d'entendre le son qu'il produit au travers de l'audio-guide accompagnant sa visite. Aujourd'hui bon nombre de musées offrent des visites multi-sensorielles. L'œil mais aussi l'ouïe et le toucher sont alors grandement sollicités quand ce n'est pas le goût ou l'odorat.

\section{La médiation corporelle}

Dans cet article je voudrais proposer un essai sur quelques formes prises par la médiation corporelle dans un musée. Par ce terme j'envisage le corps comme un outil à la fois de communication et de cognition. Il s'agira de proposer un modèle, dans le cadre du développement d'une sémiotique cognitive, dont les principales dimensions seraient les suivantes : le musée est un dispositif qui propose en creux un espace de déambulation que des corps-visiteurs sont invités à s'approprier. Chaque visiteur occupe l'espace à sa façon et, au cours de sa déambu-

1 Cf. M.JOHnSON, The body in the Mind. The bodily basis of meaning, imagination and reason, Chicago, The University of Chicago Press, 1987.

2 E. VÉRON, M. LEVASSEUR, Ethnographie de l'exposition. L'espace, le corps et le sens, Paris, Centre Georges Pompidou, 1989, p. 12 
lation, il y déploie sa gestualité opératoire. Chemin faisant, il élabore et développe - le plus souvent avec d'autres visiteurs - des stratégies cognitives grâce auxquelles il se construit une représentation des objets et thèmes abordés. Les dimensions principales de ce modèle peuvent se représenter de la manière suivante :

\section{Cognitio}

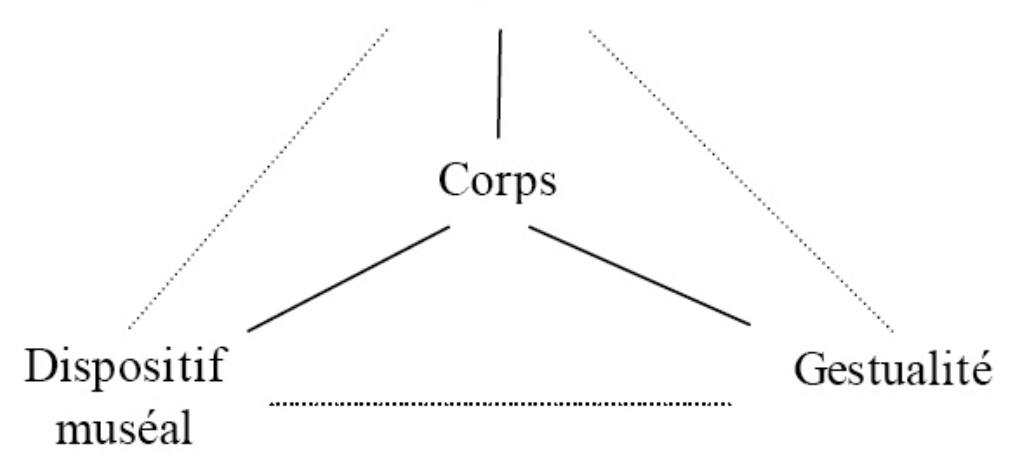

\section{Dimension cognitive}

Si l'on se place sur le plan sémiotique, c'est à Peirce que l'on doit l'idée que la communication repose fondamentalement sur le recours aux icônes. «La seule façon de communiquer directement une idée est par le moyen d'une icône ; et toute méthode indirecte pour communiquer une idée doit dépendre pour son établissement de l'utilisation d'une icône. $»^{1}$. Concrètement cela signifie que des signes qui reposeraient par exemple sur une convention - des symboles dans la terminologie de Peirce - ou sur une relation de contiguïté - des indices selon Peirce ne sont à même de communiquer quelque chose que s'ils parviennent à produire une icône dans l'esprit de l'interprète. La fameuse trilogie des signes - l'icône, l'indice, et le symbole - est donc fortement hiérarchisée dans la conception de Peirce : le symbole et l'indice impliquent toujours, d'une manière ou d'une autre, le recours à une icône pour

1 C.S. PEIRCE, Collected Papers, Harvard University Press, 2.278 traduit in G.DELEDALLE, Ecrits sur le signe, Paris, Seuil, p. 149 
fonctionner pleinement. Mais la vulgate sémiotique n'a pas retenu cette nuance importante en la diffusant. C'est à partir de travaux issus des sciences cognitives que cette idée refera son apparition dans les années 1980 sans toutefois établir de filiation avec les réflexions de Peirce. Pour Lakoff et Johnson, notre système conceptuel est de nature fondamentalement métaphorique. Ce qui signifie que nous comprenons quelque chose à partir de représentations métaphoriques dont notre système conceptuel s'est doté. Parler, discuter consistent à produire, transmettre et interpréter des métaphores. "L'essence d'une métaphore est qu'elle permet de comprendre quelque chose (et d'en faire l'expérience) en termes de quelque chose d'autre. $»^{1}$. C'est en projetant un domaine source connu sur un domaine cible non connu que la métaphore permet de comprendre et interpréter l'inconnu à partir des corrélations existantes dans un domaine source connu. Ces métaphores conceptuelles sont systématiques en ce qu'elles mettent en exergue certains éléments et en masquent d'autres. Elles peuvent prendre plusieurs formes : elles sont dites structurelles quand « un concept est métaphoriquement structuré en termes d'un autre concept », ontologiques quand elles permettent de comprendre nos expériences en termes d'objets et des substances. D'autres, au contraire, peuvent non pas structurer mais organiser un système entier de concepts les uns par rapport aux autres. En ce cas, Lakoff et Johnson parlent de métaphores d'orientation dans la mesure où elles concernent l'orientation spatiale : le haut et le bas, l'avant et l'arrière, le dedans et le dehors, etc. « ces orientations spatiales découlent du fait que nos corps sont ce qu'ils sont et se comportent comme ils le font dans notre environnement physique. Ces métaphores d'orientation donnent aux concepts une orientation spatiale. (...) De telles orientations métaphoriques ne sont pas arbitraires. Elles trouvent leur fondement dans notre expérience culturelle et physique. $»^{2}$. Les processus cognitifs qui selon ses auteurs reposent fondamentalement sur le recours aux métaphores conceptuelles, tirent leur fondement de l'expérience corporelle de l'individu et concernent essentiellement l'orientation dans l'espace. Bien sûr l'orientation ne suffit pas mais elle constitue la base expérientielle de notre entendement. À partir de ces expériences physiques fondamentales la compréhension peut développer des métaphores ontologiques ou structurales qui vont permettre des opérations

1 G.LAKOFF, M.JOHNSON, Les métaphores dans la vie quotidienne, Paris, Edition de Minuit, 1985 , p. 15

2 G.LAKOFF, M.JOHNSON, op. cit., p. 24 
mentales d'un niveau de complexité plus élevé. Notre système conceptuel est donc construit sur des concepts spatiaux qui émergent de notre interaction avec notre environnement physique. Ainsi, par exemple, le couple devant-derrière est compris à partir de l'expérience acquise au cours de nos déplacements dans l'environnement, c'est-à-dire comme orienté vers l'avant dans la mesure où notre motricité et nos différents sens sont organisés dans la direction derrière-devant. ${ }^{1}$

D'une certaine façon, cela signifie que le modèle de Lakoff et Johnson soutient l'idée que notre système cognitif est nécessairement anthropomorphe. C'est à partir et sur base de la découverte de son environnement que l'homme développe ses modes de représentation. Pour comprendre un domaine qui échappe naturellement à son expérience physique de base - l'infiniment petit comme la physique quantique ou l'infiniment grand comme l'astrophysique par exemple -, il faudra qu'il élabore des métaphores lui permettant de projeter les concepts émergents de son expérience physique sur ces domaines-cibles inaccessibles au premier abord. Autrement dit la compréhension d'un phénomène passe par une mise aux dimensions humaines de toute problématique. Et le corps joue ici un rôle déterminant. On pourrait même, en parodiant quelque peu les termes de Lévy, parler de lui comme une « technologie de l'intelligence $»^{2}$. Nous y reviendrons.

\section{Dimension gestuelle}

Longtemps confondu avec l'image (puisque comme elle il se voit), le geste n'a guère fait l'objet d'études approfondies comme si la sémiologie avait attendu d'avoir réglé le sort de l'image avant de s'attaquer à celui du geste. Les quelques travaux existants se sont perdus dans la recherche de la signification du geste et de sa grammaire. En le comparant avec le langage verbal, ils ont surtout contribué à son rejet, comme l'exprime si bien la formule "communication non verbale". Bien sûr c'était là reconnaître sa différence. Mais c'était surtout le reléguer dans un statut de tennis simple auxiliaire de l'expression verbale.

1 Ce qui n'empêche pas les auteurs d'affirmer que cette première expérience est déjà pleinement culturelle et qu'il n'y a donc pas d'"expérience physique directe" (ibidem, p. 66).

2 P.LÉVY,Les technologies de l'intelligence. L'avenir de la pensée àl'ère informatique, Paris, La Découverte, 1990. 
À nouveau ici le recours à Peirce et à sa trilogie peut être utile. Si majoritairement les images peuvent être associées aux icônes ${ }^{1}$, les gestes le seraient plutôt avec les indices c'est-à-dire ces signes qui reposent sur un rapport de contiguité contextuelle. Dans cette optique la question du sens d'un geste n'est dès lors pas à poser comme celle du sens d'un mot. Les gestes sont étroitement liés aux contextes dans lesquels ils apparaissent. C'est pourquoi une même attitude, posture ou mimique, peut prendre des sens très différents selon le contexte : ainsi en est-il des paumes orientées vers le haut du prêtre implorant son dieu ou de celles d'un animateur demandant à son auditoire de se lever. Un même geste mais des sens bien différents.

On peut soutenir que la compréhension des postures, gestes et mimiques est liée à trois grands contextes impliquant des espaces différents. Avant de les aborder il est intéressant d'observer comment Fonagy a traité la question d'un geste atypique qui ne se voit pas mais s'entend : la mimique vocale ${ }^{2}$. Par là l'auteur entend désigner l'expressivité particulière dont un locuteur fait usage quand il énonce une phrase. Cette modulation vocale, parasitaire, vient se greffer sur l'expression linguistique conventionnelle et lui attribue un sens second, analogique, qui ne se décode pas mais se comprend à partir du contexte où il est apparu. Ainsi à côté des mots utilisés par un locuteur en colère, le ton de sa voix, la prosodie empruntée, la courbe vocale déployée,... forment autant d'éléments qui vont montrer la colère en la rejouant. Pour Fonagy, la voix est un formidable outil de reproduction mimétique capable de rejouer en miniature des émotions et des expériences vécues antérieurement. Ces tendances vocales inhérentes aux émotions sont interprétées sur base de la théorie darwinienne des émotions qui considère les attitudes émotives comme des résidus d'actions ancestrales (la colère serait ainsi le produit phylogénétique du combat, la peur reproduirait la fuite,...). Il s'agirait de régressions motivées par des conditions sociales : l'interdiction oblige l'individu à renoncer à l'action directe et déclenche un processus végétatif se substituant à l'activité réelle. L'intérêt de ce travail pour notre propos réside principalement dans la description minutieuse que fait son auteur de la miniaturisation réalisée par l'appareil vocal. Au fil du temps, celui-ci a appris à rejouer en miniature des comportements qui impliquaient à l'origine une participation globale - posturale - de

1 Sans toutefois se confondre avec elles ! Cf. sur ce sujet Ph.VERHAEGEN, "Image, diagramme et métaphore" in Recherches en communication, $\mathrm{n}^{\circ} 1,1994, \mathrm{p} .19-47$

2 Y. FONAGY, La vive voix. Essais de psycho-phonétique, Paris, Payot, pp.346 
l'individu. Le corps apparaît ainsi comme un formidable outil d'assimilation et d'accommodation et en même temps comme un outil de miniaturisation des comportements. L'éclat de colère présent dans le geste vocal d'un locuteur ne propose bien sûr que quelques bribes de l'attitude posturale d'un homme en colère mais cela suffit amplement à communiquer l'émotion qui l'anime et à convoquer le contexte qui lui est d'ordinaire associé (mise sur la défensive, ripostes, ...).

Si l'on envisage la thèse de Fonagy comme prototype ou du comportement gestuel, on peut imaginer y retrouver ces deux mécanismes : d'une part, l'assimilation et l'accommodation du monde environnant par le corps propre et de l'autre, le déplacement de l'expressivité d'une partie du corps à une autre associée à sa miniaturisation. Cela conduit alors à la distinction des trois contextes que nous évoquions ci-dessus. Présentons-les brièvement.

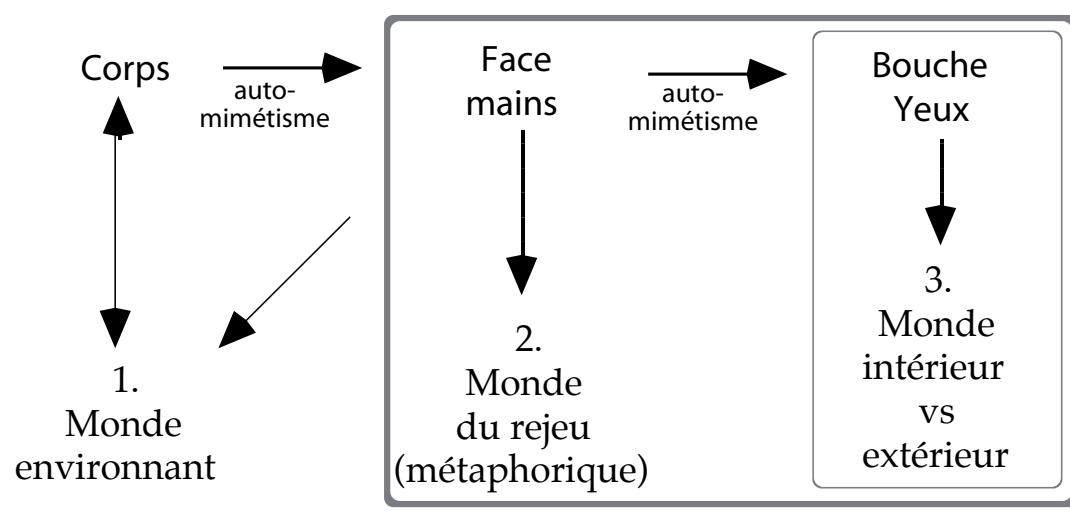

Postures

Gestes Mimiques

\section{Contexte postural}

La phylogenèse soutient d'ordinaire que le passage à la bipédie a consacré la naissance de l'Homme. En se redressant et en adoptant la marche, celui-ci est parti à la conquête de son environnement. Son comportement est le fruit de ses interactions avec celui-ci. Tout au long de son évolution, le corps de l'homme a appris à se comporter de manière adaptée. Ce que nous appelons «posture » est l'ensemble des comportements corporels globaux développés par l'espèce humaine et que chaque individu va se réapproprier au cours de son développe- 
ment personnel. Par ses postures, l'homme peut ainsi rejouer ses interactions avec le monde environnant. Il peut se mettre « à la place » de son interlocuteur, exprimer un degré d'ouverture/fermeture à l'autre, tout en restant immobile « faire ou non un pas vers » l'autre, s'orienter, mesurer la distance, " prendre la mesure » des choses, ... Les mots restent largement inappropriés pour exprimer la diversité des attitudes et des postures et les multiples nuances qui les accompagnent. Tout ici est question de degré de sens et non pas de signification. Comprendre une posture nécessite d'abord de la replacer dans son contexte d'origine et d'observer quels rapports au monde elle rejoue.

\section{Contexte gestuel}

Comme l'a si bien montré Leroi-Gourhan, l'adoption de la bipédie va de pair avec la libération de la face et des mains ${ }^{1}$. Ces dernières ont permis à l'homme de développer une activité préhensive importante l'autorisant à manipuler les objets qu'il rencontrait, à les faire tourner dans ses mains et par là, les sentir et les observer. Mais sans plus cette fois devoir se déplacer autour de l'objet pour y parvenir. Petit à petit les mains de l'homme ont permis la création d'un espace métaphorique fugace et évanescent capable de rejouer son environnement.

Pour illustrer cela, observons le mouvement circulaire des paumes, les doigts bien écartés, d'un professeur de géographie qui s'efforce de nous entretenir du système solaire. Le tracé arrondi que ses deux paumes esquissent dans l'air nous donne à voir une boule sphérique que nous identifions sans difficultés avec une planète du système solaire. Plus exactement, nous ne voyons pas, à proprement parler, de "boule sphérique", car les paumes ne font qu'en dessiner le contour. C'est notre perception qui reconstruit à partir d'indices gestuels (les deux paumes, le mouvement qu'elles décrivent, le contexte verbal) l'image de la planète en question. Un nouvel espace sert ainsi de support à la gestualité manuelle, espace qui se trouve décentré par rapport à la relation entre partenaires. C'est précisément cela que le développement du mimo-gestuel apporte au comportement postural. Autre contexte maintenant, celui d'un homme politique influent qui nous déclare qu' "il faut tenir compte de l'ensemble du problème". Le même geste donne à voir cette fois un sens plus abstrait (de globalisation, de mise en commun, de rassemblement, etc.). L'espace environnant est ainsi travaillé, sculpté

1 A. Leroi-Gourhan, Le geste et la parole, 2 tomes, Paris, Albin Michel, 1964-65 
dirions-nous, par l'orateur. Il cherche sans doute autant à "illustrer" manuellement le contenu de son discours, qu'à se rapprocher de son interlocuteur en le faisant entrer dans sa "sphère" de réflexion et/ou encore à lui montrer combien il se sent à l'aise dans le rôle qu'il joue (l' "aisance" de l'homme politique s'exprimant à la télévision, etc.). Le "même" geste illustratif produit par un autre orateur politique dans un contexte similaire pourra recevoir une interprétation plus négative. C'est que peut-être, dans ce cas-ci, l'orateur "en fait trop", ses gestes sont trop précis et trop "illustratifs": ils ne laissent pas de place dans l'interaction à l'interlocuteur, ils donnent l'impression d'avoir été "pensés". Le spectateur dans cette seconde situation ressent la "froideur", la "distance» du personnage, etc. C'est dire à nouveau combien les gestes sont une affaire de degrés et de variations subtiles. Seuls sans doute quelques gestes très stéréotypés échappent à ces principes (par exemple, le pouce levé de l'auto-stoppeur... et encore !).

Par ses mains, le "gesteur" est donc parfaitement capable de désigner l'objet dont il parle, d'en donner la forme, le mouvement, voire la structure. Plus largement, il semble que les mains peuvent remplir des fonctions pragmatiques et relationnelles étendues: elles peuvent rapprocher, éloigner, rassembler, diviser, écarter, rapprocher, élever, aplatir, soupeser, écraser,... l'objet du discours et/ou l'interactant. Rien dans le geste ne permet de distinguer sans ambiguïté ce qui renvoie à l'objet du discours, ce qui relève de l'interaction ou ce qui tient aux conditions spatio-temporelles dans lesquelles se déroulent l'interaction. Le geste rejoue tout ça d'un seul tenant. C'est pourquoi l'expression manuelle peut être rapprochée mais aussi différenciée du travail du sculpteur : les mains façonnent l'espace-même dans lequel elles se meuvent. Elles sont la matière, l'outil et le sculpteur.

\section{Contexte des mimiques faciales}

En reprenant à sa charge la fonction préhensive de la bouche, les mains ont rendu possible l'expressivité de la face. Inversement, grâce aux capacités auto-mimétiques du corps humain, la face a pu reprendre à son compte des comportements posturaux ou gestuels en les miniaturisant au niveau de l'espace buccal - le geste vocal - ou oculaire. À la différence des gestes manuels, les mimiques faciales jouent sur un rapport dehors/dedans (et ses dérivés : présence/absence, positif/ négatif). Dans le graphique ci-dessus, nous avons décrit le nouvel espace de représentation que le mimo-gestuel introduisait par rapport au comportement postural. Le regard va quelque peu le déplacer en 
l'intériorisant. C'est ce qui permet de comprendre pourquoi il est capable — comme la bouche d'ailleurs - d'absorber, de dévorer ce qu'il observe (cfr le regard vide du "fan" devant son idole, le regard du bébé, ....). Inversément, il peut extérioriser, régurgiter ce qui l'a nourrit (cfr le regard glacé de la personne en colère parfois représenté en BD par des petits poignards situés sur la trajectoire du regard). C'est ce jeu d'intériorisation versus extériorisation qui fait tout l'attrait du regard. Regarder quelqu'un les yeux dans les yeux, c'est s'autoriser à rentrer dans l'univers intérieur de l'autre, de pénétrer un peu son intimité. Le geste visuel s'articule ainsi autour d'une double direction : de l'intérieur vers l'extérieur (in-out) et vice-versa (out-in). C'est surtout le rapport entre les deux qui est déterminant : dans les interactions où la proximité prédomine, les regards "in-out" l'emportent sur ceux qui vont de l'extérieur vers l'intérieur (out-in). Dans les relations d'autorité, c'est l'inverse. L'analyse du geste vocal évoqué ci-avant a toute sa place ici. Comme le regard, il est capable de miniaturiser des comportements et des interactions et de les rejouer vocalement ${ }^{1}$.

De la posture aux gestes, le contexte de l'expressivité liée au comportement corporel passe du monde physique à l'espace de l'intériorité corporelle, un petit peu comme le sont des poupées russes.

\section{Dimension muséale}

Qu'en est-il maintenant de la portée cognitive du geste ? Qu'advient-il du geste quand celui-ci est conditionné par la mise en scène et en espace d'une exposition? Pour reprendre les termes de Véron, le concepteur d'une expo propose une mise en espace et au travers d'elle, s'expose. Le visiteur, lui, va développer des comportements d'appropriation : «visiter une exposition c'est com-poser, dans les deux sens de ce terme : celui de produire une combinatoire, et celui de s'accommoder. S'accommoder : pactiser, négocier. Visiter une exposition, c'est négocier son rapport à l'exposé (...) C'est son rapport au savoir que le sujet, par exposition inter-posée, négocie. $»^{2}$. Et cette négociation, dans le cadre du média exposition, s'appuie sur le registre de sens qu'il privi-

1 Cf. la belle description que Fonagy nous donne du/des rôle(s) joué(s) par la glotte dans l'expression de la colère, plus particulièrement dans celle de la «voix étranglée», Y. Fonagy, op. cit., p.49-50

2 E. VÉRON, M. LEVASSEUR, Ethnographie de l'exposition. L'espace, le corps et le sens, Paris, Centre Georges Pompidou, 1989, p. 21. 
légie : celui de l'indice ou encore de la métonymie et de la topologie du sens. «L'ordre métonymique opère par rapports existentiels : voisinage, partie/tout, envers/revers, contenant/contenu. Ici, les relations signifiantes s'établissent donc par des renvois indiciels. Du point de vue du sujet, le support du registre métonymique c'est son corps signifiant (...) L'exposition se constitue comme un réseau de renvois dans l'espace, temporalisé par le corps signifiant du sujet, lors de l'appropriation. $\gg^{1}$ . C'est à une première description de la médiation corporelle au sein du média exposition que nous allons maintenant nous atteler.

\section{Corps et musée}

Le dispositif muséal met en espace une thématique, mise en scène qui peu ou prou sollicite les différents contextes corporels que nous venons de présenter et par là - c'est du moins la thèse que nous soutenons - favorise certaines postures cognitives.

\section{Le monde environnant}

Le propre d'une exposition est d'étaler sa thématique dans un espace prédéfini en autant d'unités signifiantes - exhibits - que nécessaire $^{2}$. Les concepteurs ont ainsi imaginé ce que Véron appelle le «bon corps visiteur ». Le découpage de l'espace en lieux thématiques, les parcours et les noeuds décisionnels ${ }^{3}$ imaginés, les unités d'étalement élaborées, l'organisation des cimaises et des lieux de ponctuation, ${ }^{4}$, sont autant d'éléments offerts à l'appropriation du visiteur. Il importe de souligner ici que cette construction de l'espace repose plus sur un savoir intuitif, implicite et informel issu de l'expérience accumulée par les concepteurs d'expo que sur une véritable démarche de construction explicite. C'est l'analyse a posteriori qui peut mettre en évidence les

1 Ibidem, p. 24

2 J'utilise indifféremment les termes d" "unités signifiantes", d' "exhibits" ou encore d' "unités d'étalement" pour désigner chacun des dispositifs mis en place par une expo et formant en soi une entité communicationnelle homogène. Cette variabilité terminologique montre bien qu'il y a là matière à approfondissement.

3 "Un noeud décisionnel est (...) un point défini par le faisceau de directions possibles à suivre par le sujet arrivé à ce point. (Il) est vectorisé par la direction d'arrivée.", E. VÉRON, M. LEVASSEUR, op. cit., p. 50.

4 Entrées et sorties des salles, couloirs de transition d'un espace à un autre, etc. 
objectifs et les intentions qui la sous-tendent. Ce faisant, les concepteurs imaginent les différentes stratégies d'appropriation que le visiteur pourra suivre. Par la mise à plat des savoirs exposés, ils en proposent une déconstruction en unités signifiantes et attendent du visiteur qu'il procède à leur reconstruction.

Cela a pour principale conséquence de forcer le concepteur à donner à la thématique abordée des formes et des dimensions directement perceptibles par un être humain. Ainsi de cette exposition consacrée au monde enfantin dans laquelle les tables, les chaises et le mobilier avaient été ramenés proportionnellement à la taille de l'adulte. Ce dernier visitait donc l'expo en déambulant dans un espace avec les yeux d'un enfant. Ou encore ce panneau représentant graphiquement les grandes étapes de l'évolution de notre planète en ramenant celle-ci à l'échelle d'une journée terrestre.

Cet anthropomorphisme ne se limite pas à la place, aux lieux, aux grandeurs, aux distances mais aussi à l'orientation, aux parcours et aux trajectoires. Des relations comme la causalité, l'historicité ou la logique d'un phénomène, sont non seulement distribuées dans l'espace en autant d'unités signifiantes que nécessaire mais celles-ci sont articulées de telle sorte que le visiteur puisse effectuer sur elles un certain nombre d'opérations d'intellection. La stimulante distinction développée en son temps par Jacquinot pour les films didactiques ${ }^{1}$ peut être convoquée ici. Les unités d'étalement peuvent n'être reliées par aucun lien logique mais simplement données à voir - Jacquinot les appellerait des syntagmes monstratifs - ou au contraire articulées pour favoriser une opération cognitive du genre relation de cause à effet, de catégorisation, de comparaison, de dépendance - soit alors, des syntagmes démonstratifs. Ces différents syntagmes peuvent faire l'objet d'un agencement spatial - d'un montage - propre au sous-thème traité. Le recours à des notions filmiques (séquences, montage) peut poser question dans la mesure où ici le visiteur a la possibilité de déambuler à sa guise dans l'espace proposé. Ce serait oublier que la locomotion humaine effectue toujours un trajet "linéaire" même si celui-ci est fait de retours en arrière. D'ordinaire le visiteur est enclin à aller de l'avant, soit par l'organisation des lieux (un couloir bordé de cimaises n'invite pas à un retour en arrière), soit par le flux des participants qui naturellement est orienté de l'entrée vers la sortie. Cette organisation linéaire est même

1 G. JACQUINOT, Image et pédagogie, Paris, PUF, 1977, p. 77 et sv. 
parfois la seule possible. Ainsi certaines expos présentent des parcours tout à fait linéarisés où le visiteur n'a que très peu de liberté d'action en dehors du trajet imposé.

Il existe aujourd'hui dans les sciences cognitives des cadres théoriques et certaines données expérimentales qui démontrent que nos processus d'intellection s'appuient fréquemment sur des ressources matérielles disponibles dans notre environnement immédiat : des artefacts cognitifs ${ }^{1}$ conçus de toutes pièces pour nous aider à penser ou des ancres matérielles ${ }^{2}$ c'est-à-dire des éléments appartenant à notre environnement physique que nous exploitons à des fins cognitives. Les théories dites de la cognition distribuée ont élargi le rôle attribué jusque-là à la corporéité pour l'ouvrir à l'environnement matériel et social. Kirsh a ainsi montré la manière dont nous exploitons l'espace qui nous entoure ${ }^{3}$. Pour lui l'arrangement spatial des objets autour de nous fait partie intégrante de la manière dont nous pensons. En observant des cuisiniers, des gestionnaires de supermarchés ou encore des joueurs de Tetris, il a montré que ces agents faisaient preuve de ce qu'il appelle un usage intelligent de l'espace c'est-à-dire comment ils organisaient et adaptaient sans cesse leur espace de travail pour accomplir des tâches particulières. Selon Kirsh, cette gestion de l'espace permet une simplification des choix, de la perception et des processus de computation interne. Malheureusement ces études n'ont qu'un intérêt relatif pour notre propos dans la mesure où elles s'intéressent à la façon qu'a un individu d'exploiter son espace propre pour améliorer son activité cognitive. En revanche elles ne nous apprennent pas grand-chose à propos des effets d'un aménagement pré-établi de l'espace-comme dans une expo- sur le fonctionnement cognitif d'un individu-visiteur. Si la réflexion en matière de processus cognitif s'intéresse de plus en plus au rôle qu'y jouent les signes, il importe inversement de poursuivre le développement d'une véritable sémiotique cognitive.

Autre particularité à épingler : l'espace à parcourir est aussi pour le visiteur un ensemble de lieux à observer. Parmi ceux-ci, les noeuds décisionnels sont des endroits stratégiques puisque c'est à partir d'eux

1 D.A. NORMAN, "Les artefacts cognitifs" in B.Conein, N.Dodier, L.Thévenot, Raisons Pratiques, ${ }^{\circ} 4$, pp.17-34

2 E.HUTCHINS, "Material anchors for conceptual blends" in Journal of Pragmatics, 37, 2005, pp. 1555-1577

3 D.Kirsh, "The intelligent use of space" in Artificial Intelligence, 73, 1995, pp.3168 
que le visiteur va décider de la suite de son parcours et anticiper sur les choses à voir. Mais hormis ceux-ci, les lieux sont autant de terrains propices à l'observation du comportement des autres visiteurs. Nous y reviendrons.

\section{Le monde du rejeu métaphorique}

À côté de leur rôle joué dans la communication, les gestes manuels ont pour première fonction de permettre le toucher et la saisie des objets. Ils sont ainsi à la base de l'activité opérative et sensori-motrice. Ci-dessus nous avons dit des gestes de communication qu'ils mettaient en place un espace permettant à l'homme de rejouer métaphoriquement ses relations et interactions avec son environnement. Cet espace virtuel est donc un lieu de représentation métaphorique offert aussi bien à son regard qu'à celui d'autrui. Dans cet espace, l'homme peut donner une forme visuelle à ses représentations mentales et par là les observer et les analyser. Il s'agit donc d'un espace de mise à distance, d'objectivation, d'observation mais surtout peut-être un espace de simulation.

La plupart des expositions contemporaines proposent des exhibits à manipuler. Poignées à saisir, boutons à appuyer, tiroirs à ouvrir, cordes à tirer ou enrouler, puzzles à reconstituer, ... les sollicitations ne manquent pas. Nombre d'exhibit exige du visiteur un minimum d'action : il lui faut placer ou ordonner des objets sur une grille, répondre à une question en appuyant sur le bouton adéquat, etc. Pour être cognitivement intéressante, cette sollicitation fonctionnelle doit évidemment être sous-tendue par des logiques opératoires utiles à la compréhension du phénomène ${ }^{1}$.

Considérons par exemple l'exhibit suivant : cinq crânes d'espèces de dinosaures différentes - Diplodocus, Camarasaurus, Iguanodon, Chasmosaurus, Tyrannosaurus rex - sont présentés chacun dans une vitrine et identifié par un cartel. Placé sous celle-ci, un tiroir qui, une fois ouvert, laisse apparaître un moulage d'une partie de mâchoire (un tronçon de maxillaire inférieur) et un objet coupant correspondant au type de dents du dinosaure ${ }^{2}$. Le visiteur a donc la possibilité de se saisir

1 Malheureusement, il arrive encore trop souvent que seul l'aspect ludique prime. Ainsi de ces expos presse-bouton traversées par des cohortes d'enfants qui courent d'un exhibit à un autre en appuyant ici sur un bouton, là en criant dans un micro ou en tournant une manivelle.

2 Cet exhibit fait partie de la Galerie des dinosaures (salle permanente) du Museum 
de la mâchoire, de l'observer, de passer ses doigts sur la dentition et de répéter l'opération avec l'objet qui est mis à ses côtés (une pince coupante, un sécateur,...). Ce dispositif relativement simple permet aux visiteurs d'effectuer une série d'opérations d'intellection : comparer une dentition à un outil tranchant, identifier le type de dentition correspondant à chaque espèce de dinosaures, différencier les espèces sur base de leur dentition ${ }^{1}$. Aucune information particulière n'accompagne ce dispositif. Aux visiteurs d'en comprendre la logique, l'intention sous-jacente et d'en dégager les informations qui lui paraissent les plus pertinentes.

Un tel exhibit met ainsi dans les mains du visiteur une véritable technologie intellectuelle ${ }^{2}$ fait d'objets matériels (le crâne, le sécateur,...), de signes et de symboles (les informations sur le cartel), d'indices (partie de mâchoire , crâne, ...) qui sont autant à regarder qu'à palper. L'opérativité ici est première : c'est la manipulation des objets qui permettent d'enclencher les mécanismes cognitifs de comparaison. En palpant, en effleurant le tranchant ou la rugosité de la dentition, le visiteur va véritablement expérimenter la dentition et ses caractéristiques au cours d'une situation simulée. Bien évidemment le dispositif reste relativement sommaire et il faudra des contacts répétés avec d'autres exhibits pour permettre un réel approfondissement du sujet.

Une étude de Kirsh montre clairement comment nos mains interviennent quand nous pensons ${ }^{3}$. Pointer du doigt, agencer des objets sur notre plan de travail, manipuler des pions, ... peuvent permettre d'enregistrer l'état d'un processus ou simplifier la perception. En comparant deux groupes invités à comptabiliser sur base d'une photographie une configuration de pièces de monnaie, les uns autorisés à utiliser leurs mains les autres non, cette étude a montré que le groupe des «manuels » obtenaient des performances supérieures par rapport à l'autre groupe. Kirsh qualifie ces actions manuelles de stratégies complémentaires dans la mesure où elles permettent de compléter des difficultés cognitives internes en recourant à des ressources de notre environnement immé-

des sciences naturelles de Bruxelles.

1 Le dispositif favorise encore d'autres comparaisons, le cartel précisant la période concernée, le type d'alimentation du dinosaure, ...

2 J.GOODY, La raison graphique. La domestication de la pensée sauvage, Paris, Editions de Minuit,1979.

3 D.KIRSH, «Complementary strategies: Why we use our hands when we think» in J.D.MOORE \& J.F. LEHMAN (eds) Proceedings of the Seventeenth Annual Conference of the Cognitive Science Society, 1995, pp. 212-217. 
diat. Mais à nouveau ces études ne nous disent pas grand-chose sur la manière d'agencer des objets pour favoriser l'une ou l'autre opération cognitive déterminée.

\section{Le monde intérieur/extérieur}

L'évolution du comportement gestuel vers l'expressivité faciale peut être envisagée comme une démarche d'intériorisation croissante du rapport au monde, évolution conduisant au développement d'une posture cognitive dès lors plus abstraite ${ }^{1}$. Bien évidemment cette évolution va de pair avec le développement d'autres modes d'expression comme la parole, l'écriture, le dessin ou l'image. Mais la particularité du gestuel réside dans sa capacité à miniaturiser et intérioriser des comportements. La face en est sans doute le lieu principal d'expression, là où nos sens essentiels se trouvent désormais concentrés : la vue, l'ouïe, le goût et l'odorat. Chacun d'eux joue un rôle essentiel dans cette problématique d'intériorisation/extériorisation gestuelle. Compte tenu de l'espace imparti, je me limiterai ici à quelques commentaires sur la vue. Des remarques de même nature pourraient sans doute être faites pour les autres sens.

Par le regard, nous dit Brossard, "l'individu capte de l'information mais en fournit aussi simultanément. Il y a donc là un double mouvement à la fois projectif et introjectif, centripète et centrifuge du regard, puisant sa source dans et hors de l'individu $»^{2}$. Si la psychologie a largement montré la fonction du regard dans l'expression des émotions ou dans l'activité énonciative, elle a aussi souligné ses fonctions de recherche, de régulation et de contrôle des informations et montré son rôle essentiel dans la régulation de l'interaction et la structuration du groupe social (fonction socio-interactive). Aujourd'hui, on s'interroge de plus en plus sur la fonction socio-cognitive des regards.

En intériorisant le déplacement corporel, la mobilité oculaire a développé une sorte de «marche immobile à distance». Pour Véron,

1 Il en irait de même pour les interfaces développées par les nouvelles technologies, comme le fait remarquer J.L. WEISBERG, op.cit. : "l'interface informatique a constamment déplacé la frontière entre l'intérieur et l'extérieur selon un mécanisme qui schématiquement incorpore sans cesse ce qui, à l'étape antérieure se situait à l'extérieur".

2 A. BROSSARD, La psychologie du regard. De la perception visuelle aux regards, Neuchâtel, Delachaux et Niestlé, 1992, p. 9. 
le regard est un opérateur de glissement métonymique permettant à l'homme de passer d'une thématique à une autre dans un rapport de contiguité. Observer, contempler, scruter peuvent être envisagés comme des activités cognitives importantes.

Pour le saisir, revenons quelques instants à notre visiteur manipulant un exhibit portant sur la dentition des dinosaures. Ses actions peuvent servir l'apprentissage d'un autre visiteur. Celui-ci, quoique n'agissant pas directement, peut alors se nourrir des actions de l'autre. La cisaille ou le sécateur n'ont pas nécessairement besoin d'être expérimentés manuellement pour en ressentir/reconnaître la qualité du tranchant. La simple vision de ces objets familiers suffit pour s'en remémorer les caractéristiques. Le visiteur peut alors projeter les qualités de ces objets sur les morceaux de maxillaire qui sont posés à leurs côtés dans le tiroir. Cette projection permet aux visiteurs de construire un espace mixte - un blend diraient Fauconnier et Turner ${ }^{1}$ - où qualité du tranchant, type de dentition peuvent maintenant être associés à d'autres processus : le type d'alimentation, d'habitat, le mode de vie du dinosaure, ... En observant les réactions de celui qui manipule la mâchoire, le visiteur peut avoir une certaine confirmation de son interprétation (l'échange - notamment verbal - entre les protagonistes permet bien sûr de confronter les points de vue et d'approfondir le sujet).

Il est ainsi fréquent qu'un exhibit soit pris d'assaut par un groupe de personnes dont l'un manipule le dispositif tandis que les autres participent à leur manière au déroulement de l'expérience en conseillant, en avançant des explications, en posant des questions, etc. L'apprentissage collaboratif fait d'expérimentation directe, de simulation et de rejeu métaphorique est une pratique fréquente dans nos musées de sciences mais reste somme toute peu étudié2.

Il est intéressant de mentionner ici d'autres dispositifs qui sollicitent également une participation corporelle mais de manière indirecte. Le succès d'une émission comme C'est pas sorcier ${ }^{3}$ est sans doute

1 Au sens strict, un blend implique au moins quatre espaces mentaux: deux espaces d'entrée (la source et la cible), un espace générique et un espace mixte: le blend. Cfr G.FAUCONNIER, M.TURNER, The way we think. Conceptual blending and the mind's hidden complexities, New York : Basic Books, pp. 39 et sv.

2 Sur cette thématique, cf. M. NIQUETTE, "Quand les visiteurs ne sont pas seuls : l'analyse sémiocognitive", in J. EIDELMAN et M. VAN PRAËT, La muséologie des sciences et ses publics, Paris, PUF, 2000, pp. 181-198

3 Émission de vulgarisation scientifique sur France 3, produite par MFP-France Télévision. 
à chercher dans les processus décrits ci-dessus. Inséré au cœur d'un dialogue entre 4 protagonistes (Jamy, Fred, Sabine et la "petite voix"), le spectateur est invité à "manipuler des maquettes" grâce aux mains de Jamy qui nous les fait découvrir dans son laboratoire roulant. Ici c'est bien le regard posé sur le petit écran qui permet au spectateur de découvrir et de manipuler des objets par personnes interposées.

Brossard postule ainsi que le jeu des regards remplit une fonction socio-cognitive : « les fondements du savoir, de la connaissance, sont non seulement sociaux pour une part et individuels pour une autre part, mais qu'à l'intérieur de ce rapport à la connaissance, le corps mais un corps communiquant par la médiation des regards tient une place prépondérante $»^{1}$. En adaptant librement son propos, on pourrait dire que le conflit intra-individuel engendré par une observation ou une manipulation problématique entre en résonance avec un autre type de conflit, celui-là inter-individuel, conflit qui incite les partenaires à prendre conscience de l'existence de points de vue différents, c'est-àdire de visions différentes sur l'exhibit. C'est au travers notamment des échanges de regards entre les partenaires, que chacun va alors s'efforcer de trouver confirmation de son interprétation dans le regard de l'autre, ces regards constituant de la sorte un véritable lieu de confrontation sociocognitive.

\section{En guise de conclusion}

Au fil de l'évolution phylogénétique et ontogénétique, le corps est donc devenu tout à la fois un outil (se lever, marcher, prendre, ...), un instrument de communication (les postures, gestes et mimiques) et une technologie cognitive (un opérateur de glissement métonymique, de rejeu mimétique, de miniaturisation, ...). On peut véritablement parler à son propos d'une technologie intellectuelle si l'on se rappelle que Goody entendait par là une technologie - l'écriture, dans son cas - qui à la fois formait un moyen de communication et un mode de pensée. Le musée en étalant et agençant des exhibits dans un espace configuré à cette fin met à " portée de main » une problématique (scientifique et/ou sociale comme la gestion de l'eau, le recyclage ou les sources d'énergie, par exemple) que le visiteur ne pourra s'approprier qu'au travers d'un

1 A. BROSSARD, La psychologie du regard. De la perception visuelle aux regards, op. cit., pp. 246-247 
investissement corporel important. C'est cette médiation corporelle proposée par les concepteurs de l'expo et attendue des visiteurs qui fait toute la spécificité de ce média et rendra caduque toute tentative de lui substituer une visite virtuelle. Au musée, on pense d'abord avec son corps et ses mains. En ce sens il reste un important travail à mener pour mieux comprendre comment l'agencement d'indices spatiaux et corporels peut contribuer à l'amélioration de nos démarches cognitives. D'un point de vue communicationnel, c'est donc bien au développement d'une sémiotique ouverte aux questions cognitives qu'il faut s'atteler... et non l'inverse. 